\title{
PSS Parameter Optimization Based on Comprehensive Damping Effect Method Aiming at Actual Grid Characteristics in Northern China
}

\author{
Xiang-Yu Liu, ${ }^{1}$ Yu-Ling He $\mathbb{D}^{0},{ }^{2}$ Xiao-Ming Li, ${ }^{1}$ Peng Lu, ${ }^{3}$ Lun Cheng, ${ }^{3}$ and An-Chang Li ${ }^{1}$ \\ ${ }^{1}$ State Grid Hebei Electric Power Research Institute, Shijiazhuang 050021, China \\ ${ }^{2}$ Institute of Equipment Fault Diagnosis and Testing Technology, North China Electric Power University, Baoding 071003, China \\ ${ }^{3}$ State Grid Hebei Electric Power Supply Co., Ltd., Shijiazhuang 050022, China
}

Correspondence should be addressed to Yu-Ling He; heyuling1@163.com

Received 14 August 2019; Accepted 6 January 2020; Published 25 January 2020

Academic Editor: Antonio M. Gonçalves de Lima

Copyright ( 2020 Xiang-Yu Liu et al. This is an open access article distributed under the Creative Commons Attribution License, which permits unrestricted use, distribution, and reproduction in any medium, provided the original work is properly cited.

In this paper, we proposed the comprehensive damping effect method to optimize the PSS parameters. Different from other studies, the optimization in this paper is based on both the amplitude-frequency and the phase-frequency properties, while other studies mainly focus on the phase-frequency characteristics. The actual grid data in Northern China are employed to verify the optimization effect in the multimachine system. The proposed method is compared with the engineering method, the existing optimization method, and the commercial software. The comparison shows that the PSS parameters obtained by the proposed method in this paper are superior to those obtained by the engineering experience, the phase-frequency optimization method, and the commercial software. In the cases of different phase-frequency range requirements, the wider the phase-frequency range is, the better the damping effect of the obtained parameters will be.

\section{Introduction}

PSS is a simple, economical, and effective tool to improve the dynamic stability of the power system [1-4]. Due to the obvious effect, PSS now has been applied to power systems all over the world $[5,6]$. In practice, the PSS parameters usually need to be configured to achieve the satisfied performance property of a given PSS. Since the parameter setting will directly affect the PSS function and the stability level of the power grid, it is of great significance to develop advanced parameter optimizing methods for the proper parameter obtaining.

With the development of the new energy generation and the characteristic change of the power grid $[7,8]$, the engineering method for the PSS parameter setting still displays deficiencies [9]. In order to give full play of PSS, scholars studied the damping effect through the parameter optimization $[10,11]$ and the model improvement [12-14]. Some researches obtained better PSS parameters via algorithm improvement [15], while some others converted the parameter solution issue into the multiobjective optimization problem [16]. For the multimachine systems, more consideration is given to the robustness improvement [17-19], while the effectiveness of PSS parameters is not considered comprehensively at the meantime.

Generally, there are two most influential PSS models, namely, the PSS2B model $[20,21]$ and the PSS4B model [22]. The PSS2B model is the older but most widely used one, while the PSS4B model is newly proposed by IEEE in 2005. The PSS4B model provides the effective damping function in different frequency bands. However, this new PSS model does not have a good basis for the large-scale application. As addressed in Reference $[23,24]$, the fixed phase compensation with the typical parameters of PSS4B is not fit for many cases. Due to the complex structure of PSS4B and the coupling relationship among the three frequency bands, the parameter tuning of PSS4B is still difficult [13]. 
Consequently, the standard PSS4B model as well as the typical parameter settings proposed by IEEE in the year 2005 has not been widely used. By far, the PSS2A/PSS2B (the PSS2B has an extra phase-shift link, while the PSS2A does not) model is the most widely employed one in practice, while the PSS4B model is used more frequently for the theoretical investigations. In this paper, we mainly focus on the parameters of PSS2A which has been commercially applied.

The remainder of this paper is constructed as follows: the mathematical model of the proposed comprehensive damping effect method is proposed in Section 2. Then, the detailed validation as well as the effect comparison between the proposed method and other methods based on the actual running data from Northern China Grid is studied in Section 3. Finally, the main conclusions from the study are drawn up in Section 4.

\section{Mathematical Model of the Proposed Method}

2.1. Model of Accelerating Power PSS. The widely used model of accelerating power PSS is shown in Figure 1(a) [21]. This model is proposed by the IEEE standard. In practice, the commercial software employs the model illustrated in Figure 1(b), where $T_{\mathrm{r} \omega}$ and $T_{\mathrm{rp}}$ are the constants with very small values (can be practically ignored in many cases for simplification). In this paper, we employ the model indicated in Figure 1(b) and further transfer it into the form of Figure $1(\mathrm{c})$ by ignoring $T_{\mathrm{r} \omega}$ and $T_{\mathrm{rp}}$ and multiplying the former two blocks.

As indicated in Figures 1(b) and 1(c), the PSS2A PSS model has two input branches, namely, the speed input and the power input. This type structure has the advantage in restraining the influence of the power change due to the mechanical adjustment. It can also suppress the reactive power "retroregulation" effectively. The frequency properties are mainly adjusted by the phase shift, while the DC components of both the power and the rotating speed will be intercepted by the two DC blocks. At the meantime, the noise and the torsional oscillation will be removed by the notch filter.

In practical applications, the combined signal $\Delta P_{\mathrm{m}}$ of the speed branch and the power branch, as indicated in Figure 1(c), is set to zero. Then, the model is equivalent to a single input PSS. And the transfer function is

$$
G(s)=K_{\mathrm{p}} \frac{T_{\omega}}{1+T_{\omega 1} s} \cdot \frac{T_{\omega 2} s}{1+T_{\omega 2} s} \cdot \frac{1+T_{1} s}{1+T_{2} s} \cdot \frac{1+T_{3} s}{1+T_{4} s} .
$$

According to (1), the phase-frequency characteristics can be obtained and finally expressed as

$$
\begin{aligned}
\phi_{\mathrm{p}}= & -\arctan \left(\omega T_{\omega 1}\right)+\frac{\pi}{2}-\arctan \left(\omega T_{\omega 2}\right)+\arctan \left(\omega T_{1}\right) \\
& -\arctan \left(\omega T_{2}\right)+\arctan \left(\omega T_{3}\right)-\arctan \left(\omega T_{4}\right) .
\end{aligned}
$$

2.2. PSS Parameter Setting Process. According to the technical standard [20], the general process of the PSS setting is shown in Figure 2.

The primary steps are as follows:

(1) Carry out the phase-frequency characteristic experiment for the generator set without PSS compensation. In this step, the operating point of the generator should meet $P>0.8 P_{n}$ and $Q<0.2 Q_{n}$, where $P_{n}$ and $Q_{n}$ are the active power and the reactive power, respectively. This operating point is requested by the standard, more details can be found in Reference [20] and will not be repeated here due to the space limit.

(2) Calculate the phase-shift parameters according to the PSS model by experience.

(3) Judge whether the parameters meet the engineering request. If they do, they will be employed for the application, else they will be recalculated.

(4) Calculate the gain factors by the critical gain method. During the calculation, the safety margin should be taken into account.

(5) Verify the restrain effect of PSS on the local oscillation by the load step experiment or the disturbing experiment.

(6) Validate the restrain effect. The PSS parameters will be treated as qualified only when they meet the restrain effects.

In the second step of the parameter setting process, the original phase-shift parameters are calculated by experience. Such experience is generally based on the technical standard [20] and large amount of performance. More details can be found in References [25, 26].

2.3. Conventional Phase-Frequency Optimization Method. The objective of the phase-frequency optimization is to make the additional torque at $(0-2 \mathrm{~Hz})$ as close to the direction of the damping torque as possible. No matter in the local mode or the interarea mode, $0-2 \mathrm{~Hz}$ is the main frequency range for oscillations. Also, this frequency range is the very band that the PSS compensation acts on.

As shown in Figure 3, the target is to make the angle between the damper torque $M$ and the rotating difference $\Delta \omega$ close to zero in different frequency bands through the parameters adjustment. As required by the technical standard [20], the angle range between the vector $\mathbf{M}$ and the axis $\Delta \omega$ should be $\left(-20^{\circ}, 45^{\circ}\right)$. Since $-\Delta P$ is $90^{\circ}$ advanced with respect to the axis $\Delta \omega$, the angle between $\mathbf{M}$ and $-\Delta P$ should be $\left(70^{\circ}, 135^{\circ}\right)$.

As presented in Reference [15], the objective function of the phase-frequency optimization is to make the angle between $\mathbf{M}$ and $-\Delta P$ zero. The detailed objective function and the constraining conditions can be written as 


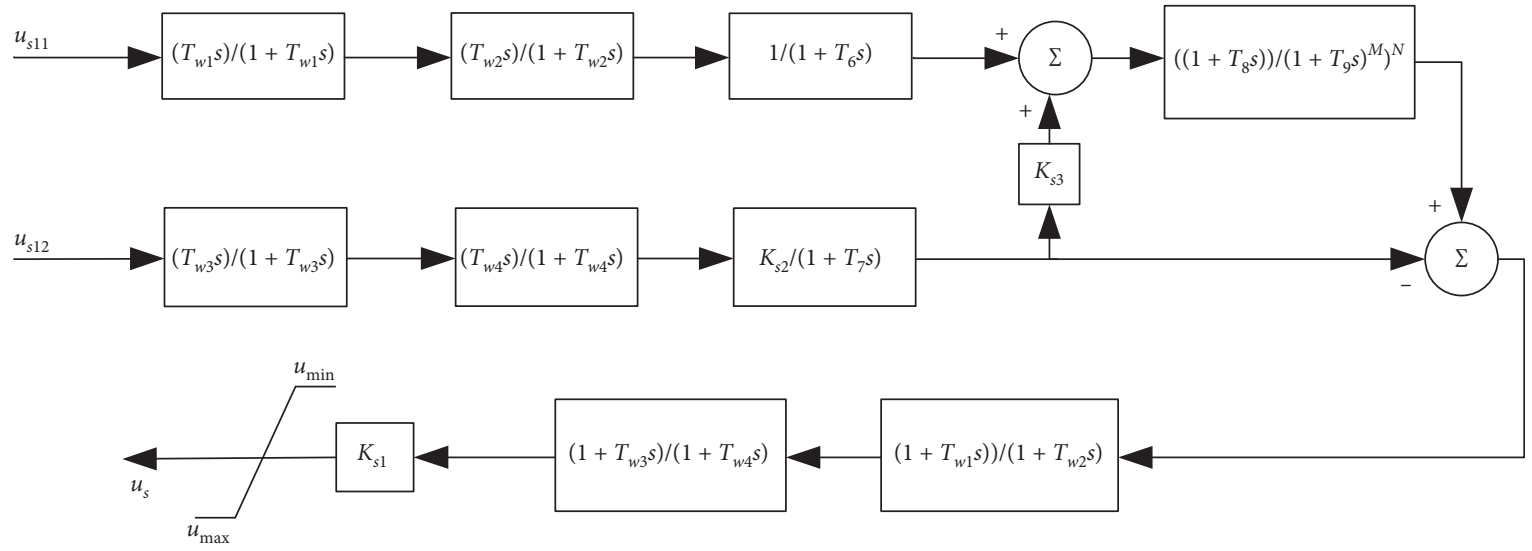

(a)
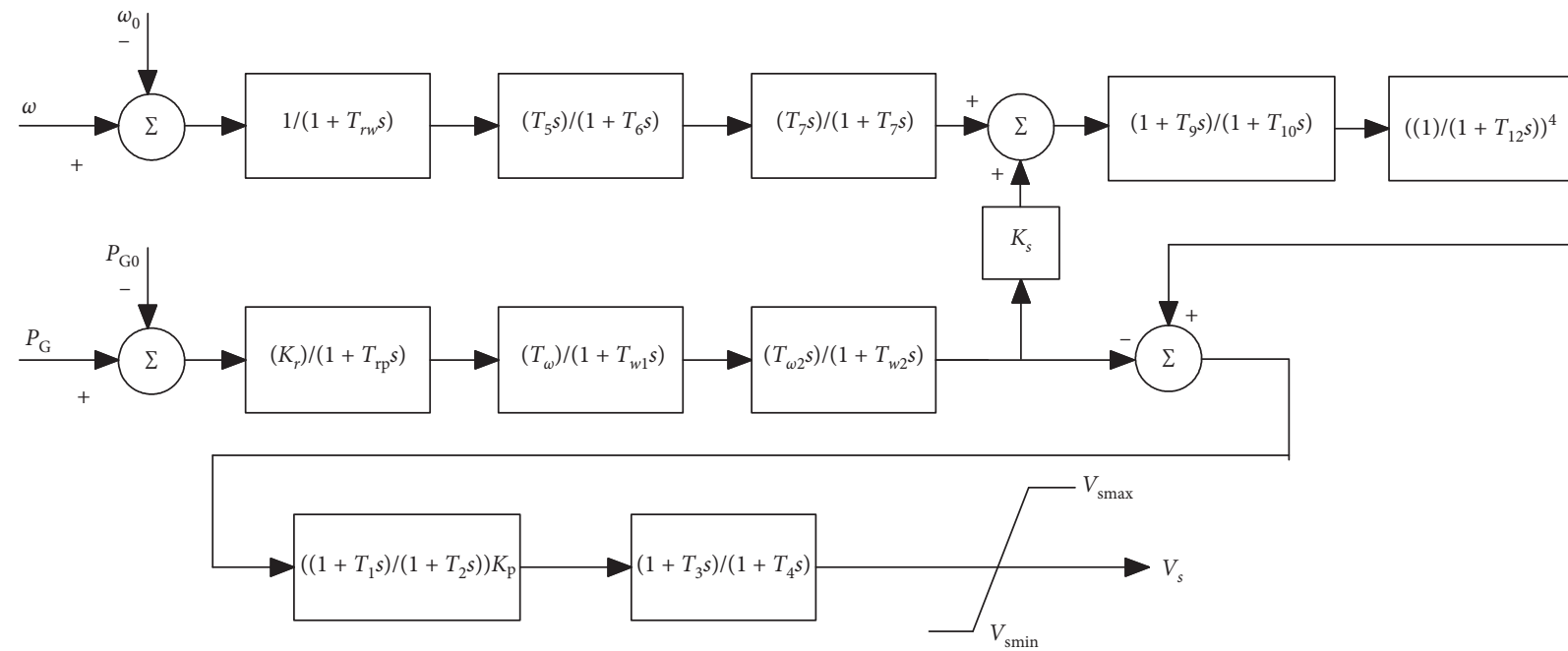

(b)
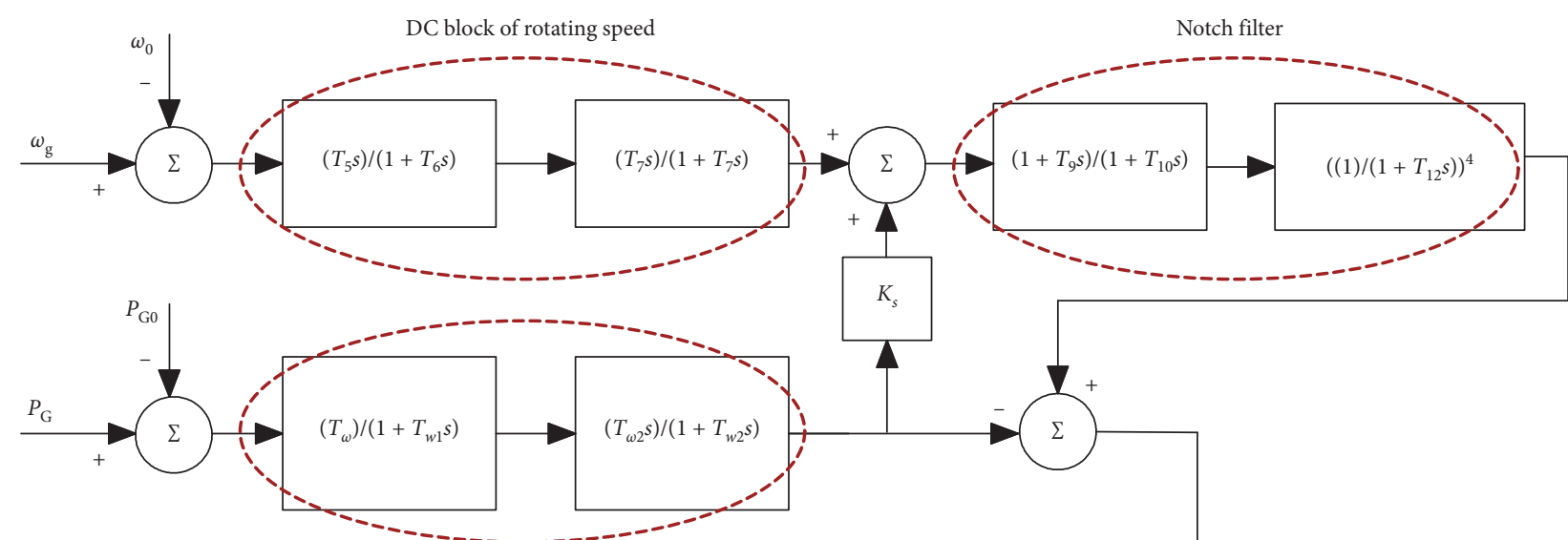

DC block of power

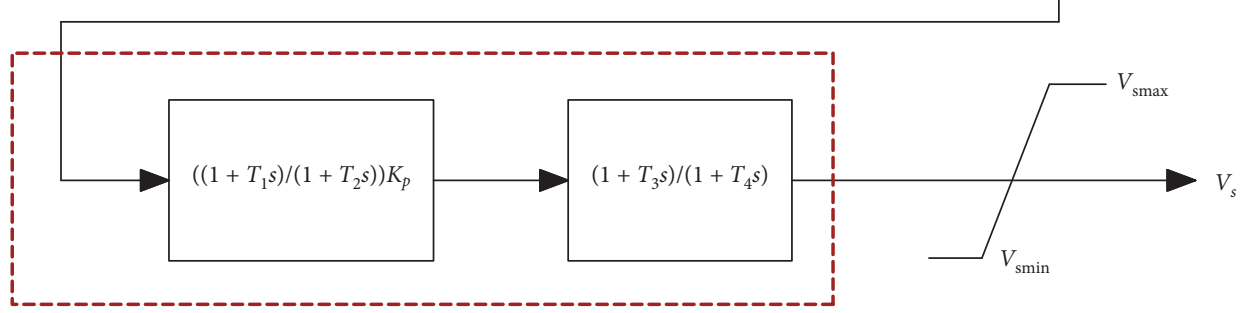

Phase shift

(c)

FIGURE 1: Block diagram of the PSS2A model based on accelerating power: (a) model in Reference [21], (b) model used in commercial software, and (c) model used in this paper. 


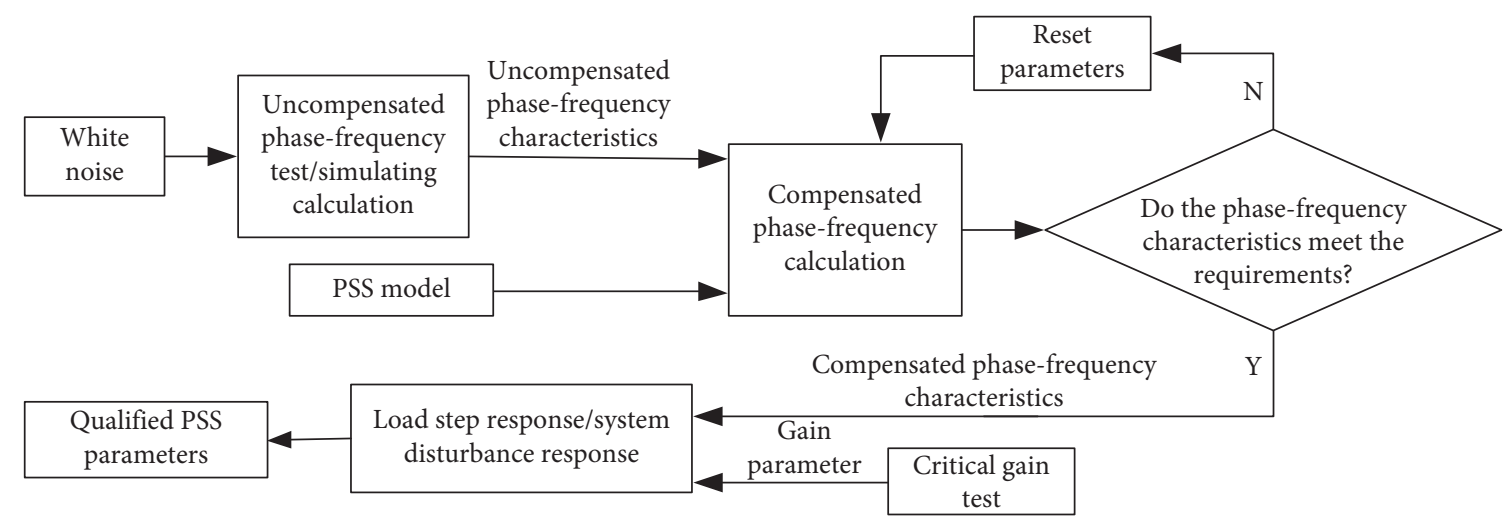

FIGURE 2: Flowchart of PSS parameter setting.

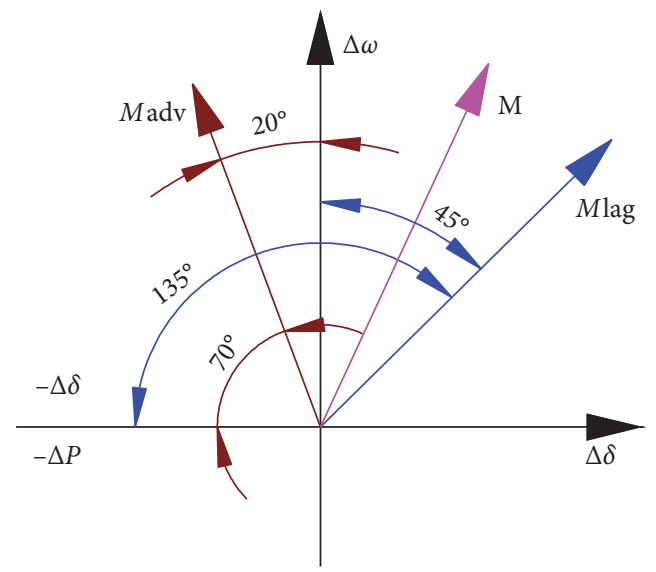

Figure 3: Vector diagram of the input signal and phase compensation.

$$
\begin{cases}\min & \sum_{j=1}^{m}\left(\varphi_{0}\left(f_{j}\right)+\varphi_{\mathrm{p}}\left(f_{j}\right)+90\right)^{2} \\ & -135<\varphi_{0}\left(f_{j}\right)+\varphi_{\mathrm{p}}\left(f_{j}\right)<-70, \quad f_{j} \in(0.2,2) \\ \text { s.t. } & -135<\varphi_{0}\left(f_{j}\right)+\varphi_{\mathrm{p}}\left(f_{j}\right)<-50, \quad f_{j} \in(0,0.2] \\ & 0 \leq T_{i} \leq 1, \quad i=1,2,3,4,\end{cases}
$$

where $m$ is the number of the probe point at $0 \sim 2 \mathrm{~Hz}$ (usually the point step is $0.1 \mathrm{~Hz}$, and there are 20 points in total), $\varphi_{0}\left(f_{j}\right)$ is the uncompensated phase-lag angle of the generator at $f_{j}, \varphi_{\mathrm{p}}\left(f_{j}\right)$ is the compensated phase angle, $T_{\omega 2}$ and $T_{\omega 1}$ are the constants set by experience, and $T_{1} \sim T_{4}$ are the optimization objects in $[0.01,1] . \varphi_{0}\left(f_{j}\right)$ can be measured directly, while $\varphi_{\mathrm{p}}\left(f_{j}\right)$ depends on the parameters selection. $T_{\omega 2}$ and $T_{\omega 1}$ are usually preset as constants.

Actually, the phase-frequency optimization is to get the most adaptable parameters. The advantage is that the parameter acquisition can be more explicit than the usual engineering methods.
2.4. Comprehensive Damping Effect Optimization Method. Primarily, the PSS parameter setting and optimization are based on the technical standard [20], which mainly focuses on the phase-frequency optimization. As mentioned in $[25,26]$, among the optimization means, the phase-frequency method is comparatively more robust. This method is therefore employed as the major method for the PSS parameter optimization. However, if a more satisfied optimizing effect is requested over the limit of the phase-frequency method, the comprehensive damping effect optimizing method, which takes into account both the phase-frequency and the amplitude-frequency optimization, should be further taken into account. In this paper, we carry out such optimization to improve the comprehensive damping effect.

As an improvement to Section 2.3, the maximum damping force, which includes both the phase-frequency characteristics and the amplitude-frequency properties, is taken as the optimization objective in this paper. The parameter optimization model is shown in (4). The optimization objective is the maximum sum of the damping forces obtained in the target frequency range $(0-2 \mathrm{~Hz})$ : 


$$
\begin{cases}\max & \sum_{j=1}^{m} T_{t}^{\prime}\left(f_{j}\right) \\ & -135<\varphi_{0}\left(f_{j}\right)+\varphi_{\mathrm{p}}\left(f_{j}\right)<-70, \quad f_{j} \in(0.2,2) \\ \text { s.t. } & -135<\varphi_{0}\left(f_{j}\right)+\varphi_{\mathrm{p}}\left(f_{j}\right)<-50, \quad f_{j} \in(0,0.2] \\ & 0 \leq T_{i} \leq 1, \quad i=1,2,3,4,\end{cases}
$$

where

$$
\left\{\begin{array}{l}
T_{t}^{\prime}=\frac{T_{t}}{T_{\omega} K_{\mathrm{p}} F_{0}}, \\
T_{t}=F_{0} \cdot F_{\mathrm{p}} \cdot \sin \left[\varphi_{0}\left(f_{j}\right)+\varphi_{\mathrm{p}}\left(f_{j}\right)\right], \\
F_{\mathrm{p}}=\frac{K_{\mathrm{p}} \cdot T_{\omega}}{\sqrt{1+\left(T_{\omega 1} \cdot \omega\right)^{2}}} \cdot \frac{T_{\omega 2} \cdot \omega}{\sqrt{1+\left(T_{\omega 2} \cdot \omega\right)^{2}}} \cdot \frac{\sqrt{1+\left(T_{1} \cdot \omega\right)^{2}}}{\sqrt{1+\left(T_{2} \cdot \omega\right)^{2}}} \cdot \frac{\sqrt{1+\left(T_{3} \cdot \omega\right)^{2}}}{\sqrt{1+\left(T_{4} \cdot \omega\right)^{2}}},
\end{array}\right.
$$

where $F_{0}$ denotes the amplitude-frequency property and usually is a constant which depends on the exciting system of the generator system, $T_{\omega}=T_{\omega 1} / M$ is also a constant which needs to be calculated in advance of the optimization, $F_{\mathrm{p}}$ is the parameter to indicate the amplitude-frequency, and $K_{\mathrm{p}}$ is the constant gain factor.

As indicated in equations (4) and (5), both the phasefrequency characteristics and the amplitude-frequency properties are involved in the object function. That means the optimization needs to take into both the phasefrequency and the amplitude-frequency properties. In equation (4), the constraining conditions are the requested items defined by the standard [20]. These constrain conditions are set to ensure the positive direction of the vector $\mathbf{M}$. By keeping the vector $\mathbf{M}$ in the positive direction, the angle error of the moment $T_{t}$ can be avoided so that the damping effect can be kept on a satisfied level. In the range of $0-0.2 \mathrm{~Hz}$, the additional torque $\mathbf{M}$ should vary between advance $\Delta \omega 40^{\circ}$ and lag $\Delta \omega 45^{\circ}$. However, in the range of $0.2-2 \mathrm{~Hz}$, the additional torque $\mathbf{M}$ should vary between advance $\Delta \omega 20^{\circ}$ and lag $\Delta \omega 45^{\circ} . T_{\omega 2}$ and $T_{\omega 1}$ are preset as constants, and the phase-shift link coefficients $T_{1} \sim T_{4}$ are taken as the targets to be optimized, whose value range can be set to $[0.01,1]$. Actually, equation (5) describes the problem of obtaining the maximum damping effect under the specified phase-frequency characteristic requirements. By solving this problem, the best damping effect can be obtained within the specified adaptive range. At the meantime, the balance between the adaptability and the effectiveness can be achieved as well.

\section{Analysis on Optimization Results}

3.1. Optimization of Damping Effect Based on Grid Characteristics. The current standard such as [20-22] employs the ideally the theoretical model, for instance, the IEEE 10-bus system model, for the evaluation. Many scholars carried out detailed studies on these theoretical models, for instance, References [27-29] are the typical ones. However, the actual system, for example, the Chinese grid which combines large amount of generators and subnetworks, could be far more complex than the ideal model. The difference between the standard theoretical model and the actual situation is that the characteristics of the systems without compensation are varied. This kind of difference is actually the diversity in the constraining conditions, while it should not affect the similar qualitative result. In this paper, specifically aiming at the Chinese grid characteristics, we mainly focus on the PSS property improvement by using the actual running data and will not take the ideally theoretical model as the study object.

A generator unit in the actual power grid is selected as the research object. This unit locates in Cangdong Power Plant, which is under the charge of State Grid Hebei Electric Power Company. The uncompensated phasefrequency characteristics of this unit are obtained through PSD Power Tools (widely used commercial software in China), as shown in Figure 4. The simulation adopts the offline data of the actual power grid, namely, the Northern China Grid, where the unit is located. When the PSS of the unit is out of operation, it shows that the damping ratio of the power oscillation curve is 0.0488 and the oscillation frequency is $1.2471 \mathrm{~Hz}$ if a fault occurs in the power grid.

According to the uncompensated phase-frequency characteristics based on the actual power grid, the PSS parameters $\left(T_{1} \sim T_{4}\right)$ are solved according to the models described in Sections 2.3 and 2.4, respectively. The parameters obtained by different methods and the corresponding damping ratios for the same faults are shown in Table 1 . The phase-frequency characteristics with compensation are shown in Figure 5, and the damping force characteristics are shown in Figure 6. The power 


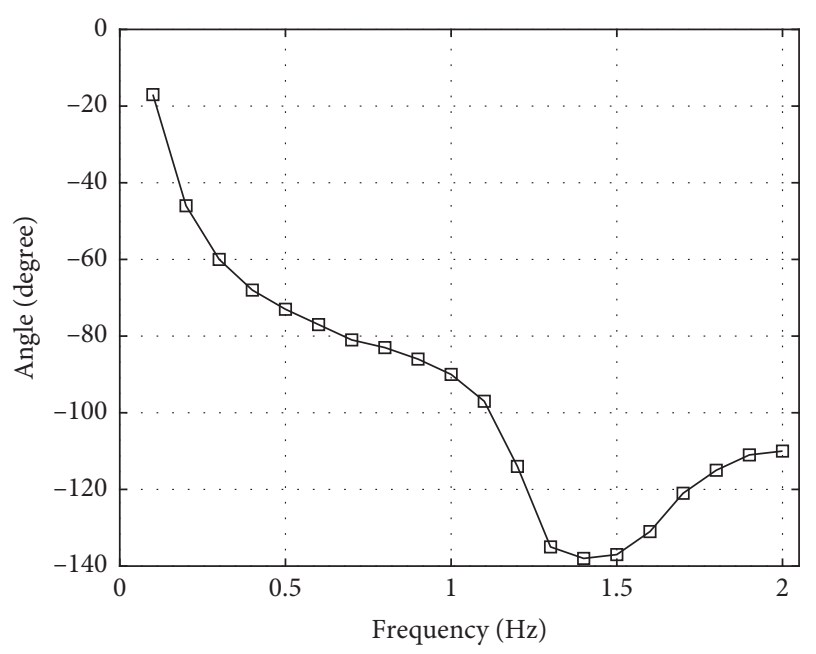

FIgURE 4: Phase-frequency characteristic curves without compensation obtained by actual power grid simulation.

TABLE 1: PSS parameters of different methods (unit: 1).

\begin{tabular}{lccccc}
\hline Methods & $T_{1}$ & $T_{2}$ & $T_{3}$ & $T_{4}$ & $\begin{array}{c}\text { Damping } \\
\text { ratio }\end{array}$ \\
\hline $\begin{array}{l}\text { Engineering experience } \\
\begin{array}{l}\text { Phase-frequency } \\
\text { optimization }\end{array}\end{array}$ & 0.21 & 0.02 & 0.3 & 0.03 & 0.1102 \\
$\begin{array}{l}\text { Damping force } \\
\text { optimization }\end{array}$ & 0.21 & 0.01 & 0.22 & 0.01 & 0.1003 \\
& 0.31 & 0.05 & 0.59 & 0.06 & 0.1775
\end{tabular}

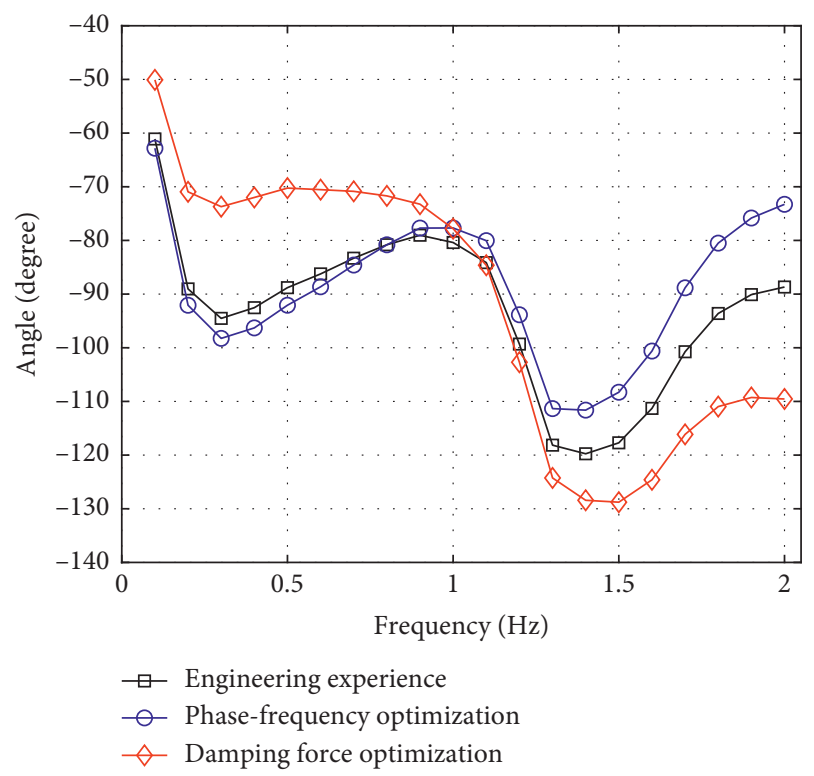

FIGURE 5: Phase-frequency characteristic curves with compensation obtained by different methods.

oscillation curves of the unit for the same fault with different PSS parameters are shown in Figure 7.

In Figure 5, it shows that the phase-frequency characteristics obtained by the phase-frequency optimization method and the engineering experience method are quite

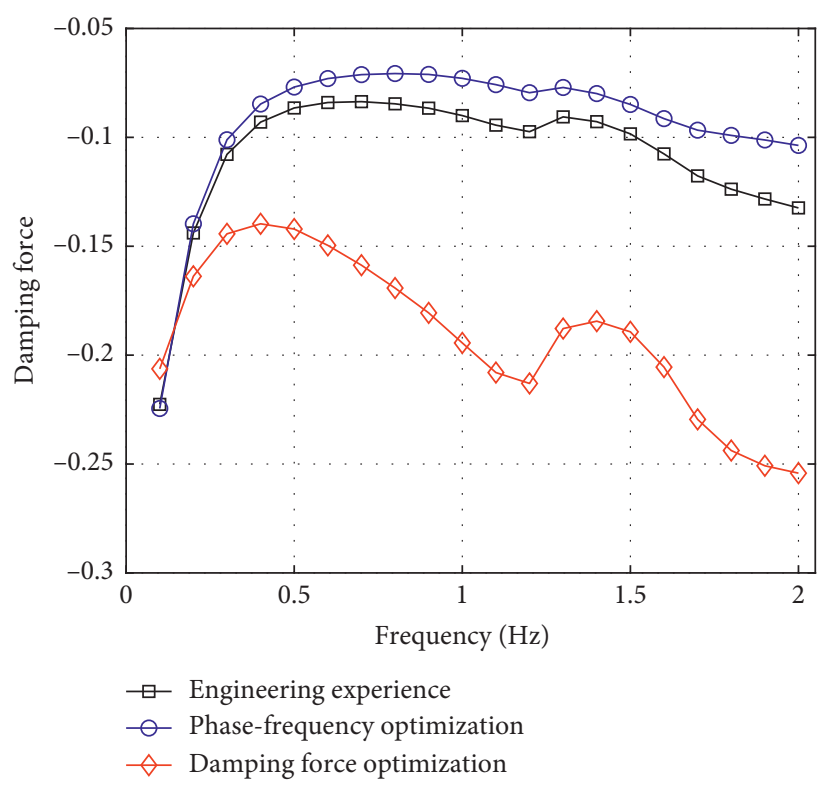

FIguRE 6: Damping force characteristic curves obtained by different methods.

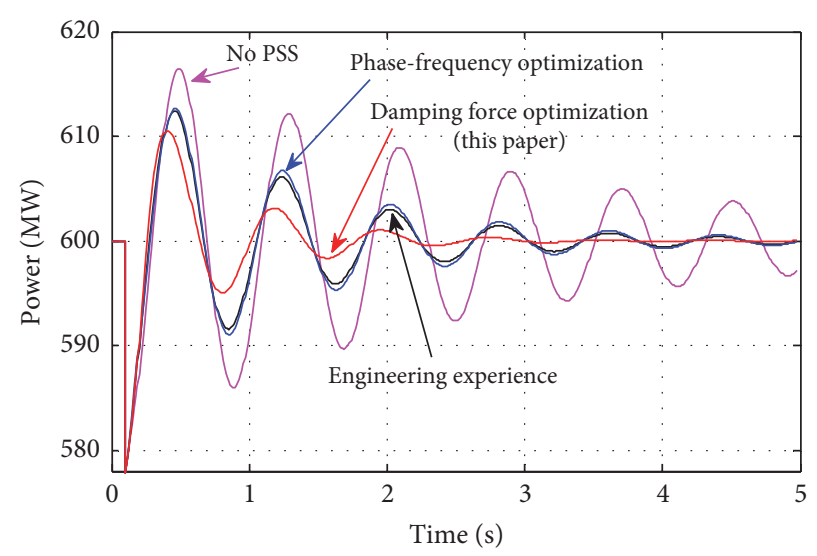

FIgURE 7: Power oscillation curves with different PSS parameters obtained by varied methods.

similar, while the result obtained by the comprehensive damping force method has a larger change. Comparing Figure 5 with Figure 6, it can be seen that a better damping effect will be obtained under the condition that the requirements for phase-frequency characteristics are not so strict. As illustrated in Figure 6, the comprehensive damping force method proposed in this paper is able to provide a greater damping effect within the frequency range of $0.2 \sim 2 \mathrm{~Hz}$. One thing that needs specific note is that the proposed method provides a little less damping force at $0.1 \mathrm{~Hz}$. Considering that the general frequencies of the lowfrequency oscillation are above $0.2 \mathrm{~Hz}$ [30], the damping force optimization method will therefore achieve a better effect.

As suggested from Table 1, the damping ratio of the power oscillation obtained by the comprehensive damping force optimization method is the maximum, while the phase-frequency optimization method has the minimum 
ratio. According to Figure 7, by the phase-frequency optimization parameters or the engineering parameters, the power oscillation of the generator unit needs about 6 oscillations to get stable again. However, using the comprehensive damping force method proposed in this paper, it only needs about 3 oscillations, and the amplitude of the damping force is obviously smaller.

\subsection{Comparison with Commercial Software Parameters.} In order to fully study the practical effect of the proposed method, the commercial software which is widely used in China is introduced as a reference. The parameters of the phase-shift link are calculated by both the commercial software and the method proposed in this paper, respectively. Here we keep the three parameters $K_{\mathrm{p}}, T_{\omega 2}$, and $T_{\omega 1}$ the same but just adjust the target optimizing parameters so that the influence of these parameters can be avoided. Consequently, the impact of the target parameter optimization on the PSS effect can be accurately obtained and compared. This type of parameter setting method is proposed in Reference [20].

The PSS parameters obtained by different methods and the damping ratios are shown in Table 2. The comparison of the phase-frequency characteristics is shown in Figure 8, and the comparison of the damping force characteristics is shown in Figure 9. The power oscillation curves of the generator unit under the same fault are shown in Figure 10.

It is shown in Figure 8 that the phase-frequency characteristic curve obtained by the commercial software is closer to $-90^{\circ}$ than the proposed method. Both of the curves are within the range required by the technical standards. The commercial software has a better phase-frequency characteristic, while the method in this paper has a better damping effect. Therefore, the damping effect of PSS can be increased by relaxing the phase-frequency characteristics to a certain extent.

It can be seen from Figure 9 that the comprehensive damping force optimization method proposed in this paper is able to provide a greater damping force within the frequency range of $0.1 \sim 2 \mathrm{~Hz}$.

According to Table 2 and Figure 10, the damping ratio obtained by the method proposed in this paper is obviously higher than that obtained by the commercial software. The commercial software needs about 4 oscillations to return stable, while the method in this paper only needs about 2 oscillations. Moreover, the oscillation amplitude obtained by the proposed method is obviously smaller than that obtained by the commercial software. Such result further verifies that the proposed method gains a better damping effect.

\subsection{Comparison of Different Phase-Frequency Range} Requirements. Considering that the expansion of the phasefrequency range may increase the damping effect, we investigate the relation between the damping effect and the requirements of phase-frequency range in this section. Three sets of PSS parameters are obtained in different phase-frequency ranges by using the proposed method. The detailed frequency ranges, the damping ratios, and the corresponding PSS parameters are shown in Table 3. The damping ratios of
TABLE 2: Parameters obtained by the commercial software and the proposed method (unit: 1).

\begin{tabular}{lccccc}
\hline Methods & $T_{1}$ & $T_{2}$ & $T_{3}$ & $T_{4}$ & $\begin{array}{c}\text { Damping } \\
\text { ratio }\end{array}$ \\
\hline $\begin{array}{l}\text { Commercial software } \\
\begin{array}{l}\text { Damping force } \\
\text { optimization }\end{array}\end{array}$ & 0.19 & 0.02 & 0.3 & 0.03 & 0.1452 \\
\hline
\end{tabular}

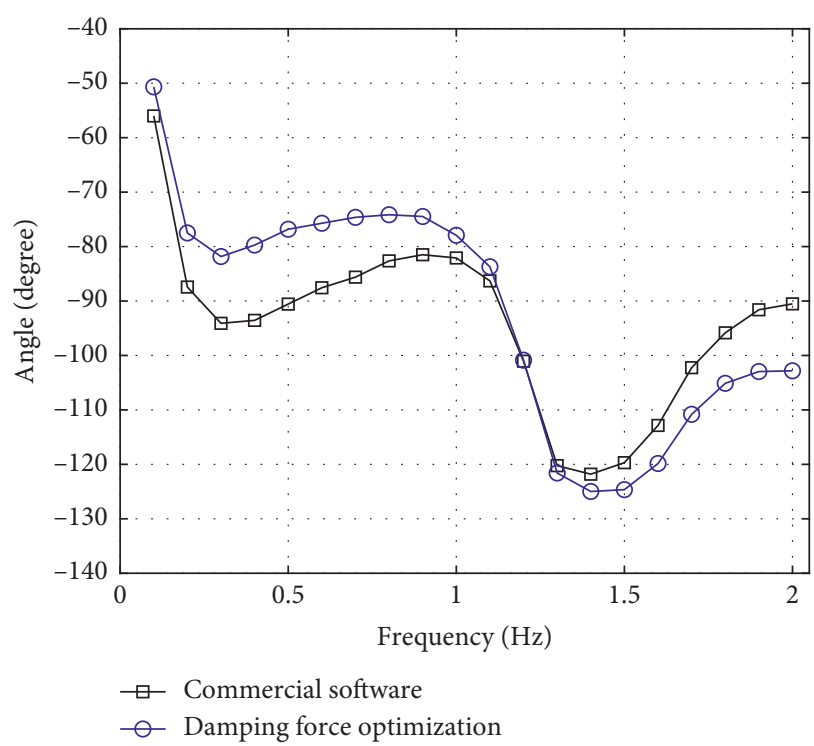

Figure 8: Phase-frequency characteristic curves with compensation obtained by the commercial software and the proposed method.

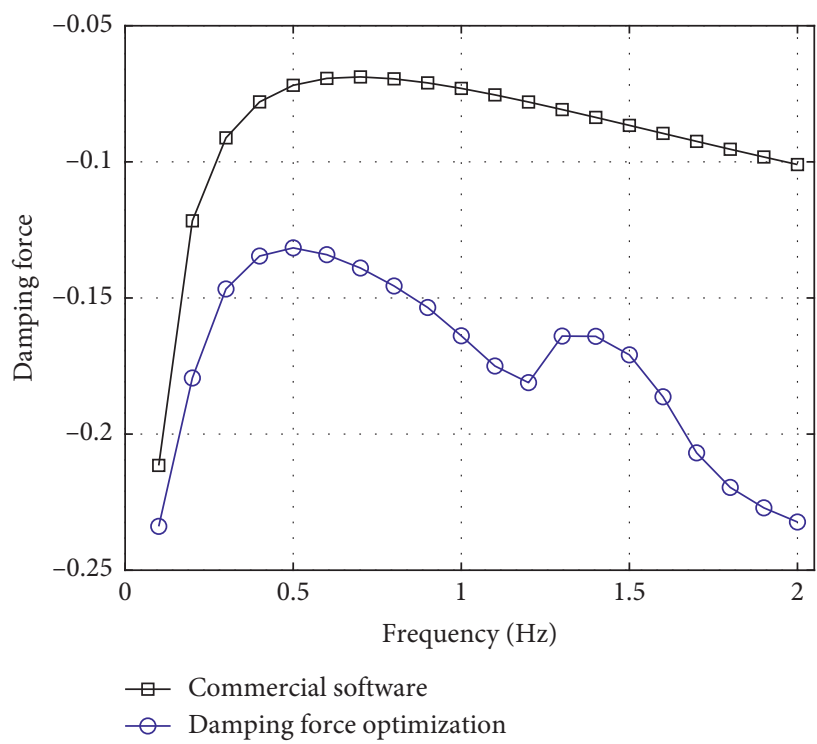

FIgURE 9: Damping force characteristic curves obtained by the commercial software and the proposed method.

different parameter sets are obtained under the same fault. The phase-frequency characteristics obtained by different parameter sets are shown in Figure 11, while the damping force characteristics are shown in Figure 12. 


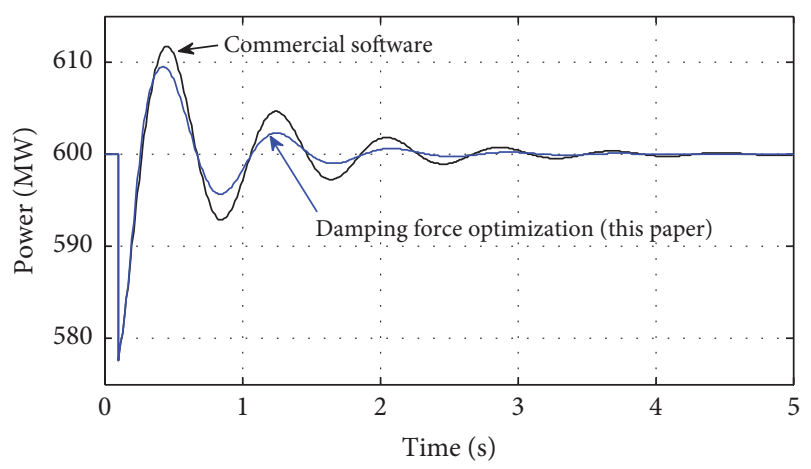

Figure 10: Power oscillation curves calculated by the commercial software and proposed method.

TABLE 3: PSS parameters of different phase-frequency ranges (unit: degree for phase-frequency range and 1 for others).

\begin{tabular}{lccccc}
\hline Phase-frequency range & $T_{1}$ & $T_{2}$ & $T_{3}$ & $T_{4}$ & Damping ratio \\
\hline-70 to -135 & 0.31 & 0.05 & 0.59 & 0.06 & 0.1775 \\
-75 to -135 & 0.2 & 0.04 & 0.69 & 0.05 & 0.1604 \\
-80 to -135 & 0.15 & 0.04 & 0.7 & 0.04 & 0.1395
\end{tabular}

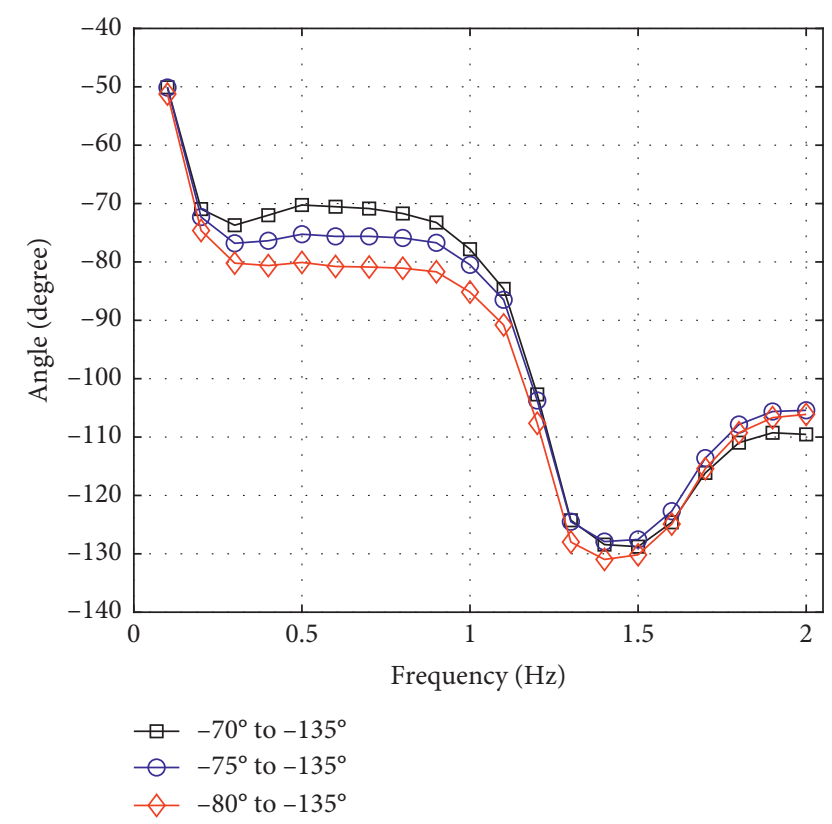

FIgURE 11: Phase-frequency characteristic curves with compensation in different phase-frequency ranges.

Comparing Figure 11 with Figure 12 as well as Table 3, it can be seen that the increase of the phase-frequency range will lead to the greater damping force. That means the wider phase range can provide the better damping properties. As is suggested from Table 3 and Figure 12, the phase-frequency characteristics and the damping force properties conflict with each other. They both usually cannot be optimal at the same time. When the phase-frequency characteristic is set closer to $-90^{\circ}$, the damping effect will be reduced. Therefore, in the practical application, these two

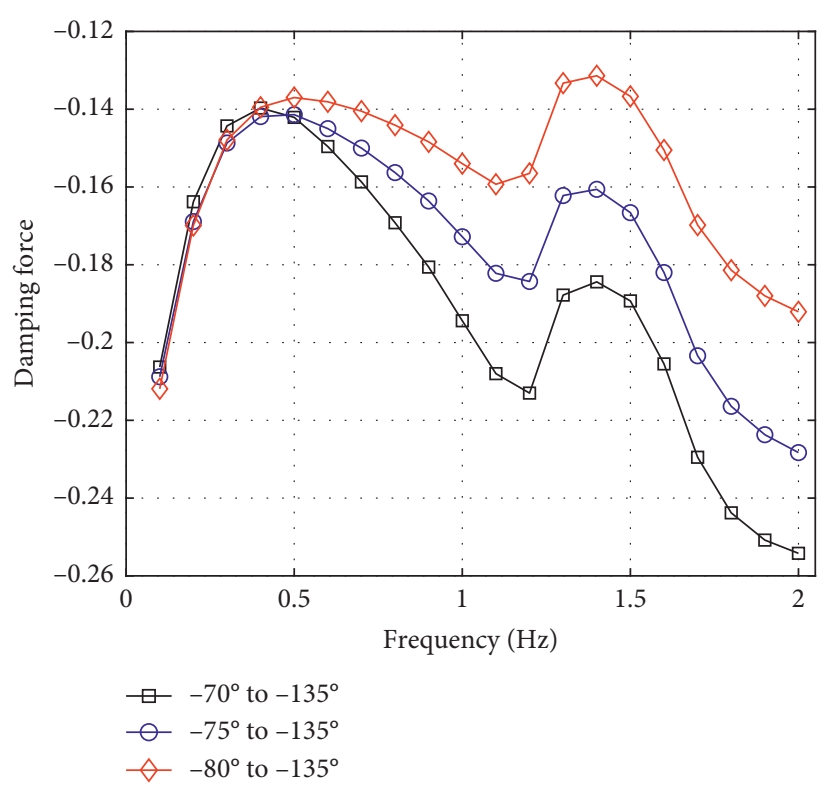

FIGURE 12: Damping force characteristic curves in different frequency ranges.

indexes should be balanced according to the actual situation to achieve the coordination of both the adaptability and the effectiveness.

3.4. Gain Coefficient Analysis. Given that the gain coefficient is another important part of PSS parameter setting, it is a more scientific way to compare the damping effect by considering the gain coefficient setting. It is found that the PSS parameters are closely related to the critical point of the high-frequency gain [23]. The gain values obtained by the commercial software and the proposed method (frequency range $-80^{\circ}$ to $-135^{\circ}$ ) are shown in Figure 13. The highest values of the high-frequency gains are 0.2307 and 0.2274 , respectively. According to [23], the intrinsic gain coefficient of the generator excitation system is recorded as $K_{0}$, and the critical gain is calculated as

$$
\begin{aligned}
K_{\text {Critical }} & =K_{0} \cdot T_{\omega} \cdot K_{\mathrm{p}}^{\mathrm{com}} \cdot K_{\max }^{\mathrm{com}} \\
& =K_{0} \cdot T_{\omega} \cdot K_{\mathrm{p}}^{\mathrm{pro}} \cdot K_{\max }^{\mathrm{pro}},
\end{aligned}
$$

where $K_{\max }^{\text {com }}$ denotes the maximum amplitude gain obtained by the commercial software $\left(K_{\mathrm{p}}=1\right), K_{\max }^{\text {pro denotes the }}$ largest amplitude gain obtained by the proposed method, $K_{\mathrm{p}}^{\text {com }}$ denotes the max gain (see $K_{\mathrm{p}}$ in Figure 1) that could be obtained by the commercial software, and $K_{\mathrm{p}}^{\text {pro }}$ denotes the max gain (see $K_{\mathrm{p}}$ in Figure 1) that could be obtained by the proposed method.

Then there is

$$
\begin{aligned}
\frac{K_{\mathrm{p}}^{\mathrm{pro}}}{K_{\mathrm{p}}^{\mathrm{com}}} & =\frac{K_{\max }^{\mathrm{com}}}{K_{\max }^{\mathrm{pro}}} \\
& =\frac{0.2307}{0.2274}>1,
\end{aligned}
$$

which means 


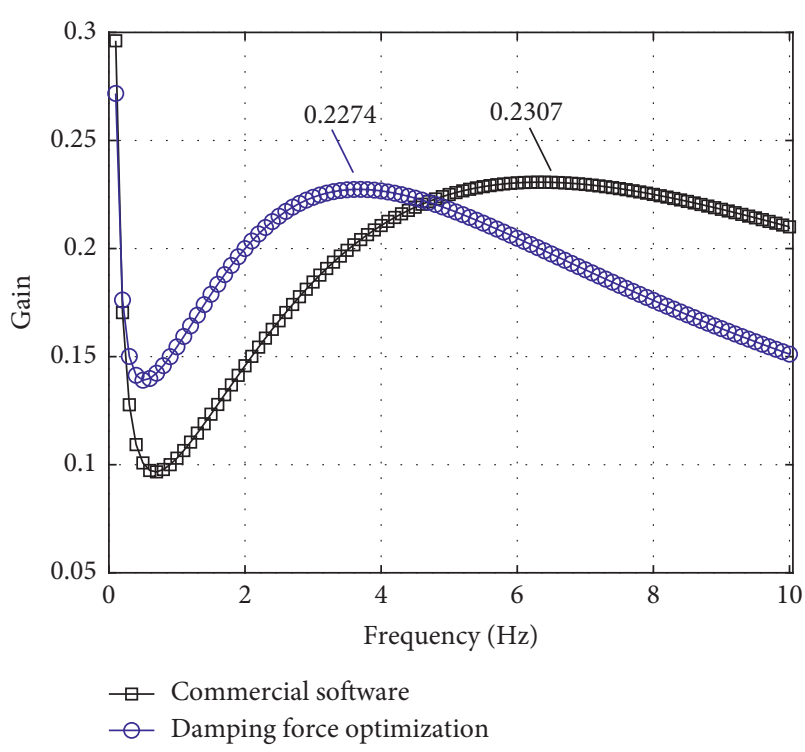

FIGURE 13: Gain curves obtained by the commercial software and the proposed method.

$$
K_{\mathrm{p}}^{\mathrm{pro}}>K_{\mathrm{p}}^{\mathrm{com}}
$$

In other words, the parameters obtained by the proposed method can be adjusted to a larger gain coefficient than those obtained by the commercial software. As shown in Figure 14, since the proposed method can obtain a greater damping force under the same gain coefficient, its advantage will be more obvious under the larger gain coefficient conditions. Therefore, the proposed method is able to optimize the parameters for a better damping effect.

\subsection{Analysis on Actual Application Effect in Multimachine} Power Grid. In order to test the effect of the proposed method in multimachine system with actual measured data, a regional power grid is selected as the research object. This power grid is named Mengxi Grid. It is the third largest power grid in China but only locates in the west of the Inner Mongolia. This regional grid has an inherent natural oscillation mode with the Northern China Grid.

As shown in Figure 15, the transmission power of the regional grid to the main network is limited by the dynamic stability. By the small disturbance analysis, it is concluded that the regional power grid has an oscillation mode with a damping ratio of 0.069 and an oscillation frequency of $0.4 \mathrm{~Hz}$. A total of 112 generator units in the regional power grid have a participation rate which is greater than 0.05 (indicates strongly correlated) in this oscillation mode.

Ten of the 112 generator units are selected, and their PSS parameters are optimized by the proposed method. After the parameters are changed, the oscillation mode of the regional grid will be also different. The comparison between the regional grid oscillation modes before and after optimization is shown in Table 4. After parameter optimization, the damping ratio of the oscillation mode becomes 0.078 , which is $13 \%$ higher than the value before change.

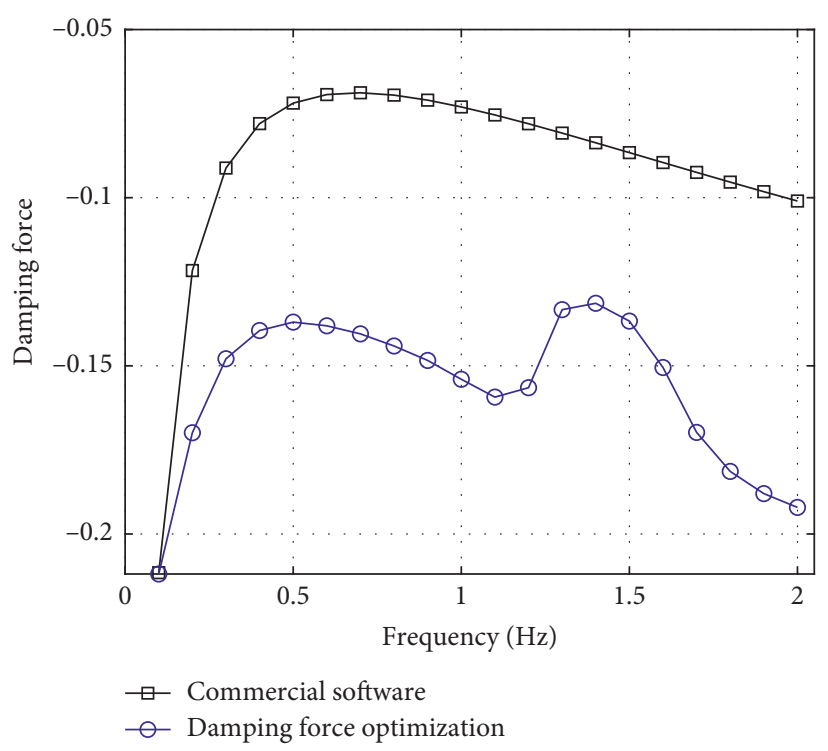

FIgURE 14: Damping force characteristic curves with the same gain coefficient obtained by the commercial software and the proposed method.

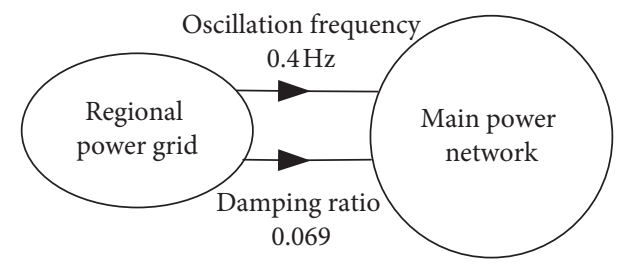

FIGURE 15: Schematic diagram of the grid structure.

Therefore, the proposed method is able to provide a better damping effect in multimachine system in actual applications. And the proposed method shows its potential engineering application value.

3.6. Suggestions for Optimization of Power Grid Dynamic Characteristics. As previously studied, taking the optimal damping force as the optimization objective can obtain a better damping effect. In order to optimize the dynamic characteristics of the power grid and improve the transmission capacity of the tie line, the PSS parameters can be optimized by the method of this paper. Based on the study of this paper as well as the practical parameter setting process (Section 2.2), the PSS parameter optimization process is suggested to be performed as shown in Figure 16.

The process can be described in the following steps:

(1) Obtain the dynamic characteristics of the power grid by simulation analysis

(2) Select the key generator units to be optimized for the natural oscillation mode of the power grid

(3) Establish the optimization targets for the selected generator units, and specify its acceptable phasefrequency characteristic requirements 
TABLE 4: Oscillation mode comparison of the regional power grid.

\begin{tabular}{lccc}
\hline Mode & Eigenvalue (unit: 1) & Frequency (Hz) & Damping ratio (unit: 1$)$ \\
\hline Original parameters & $-0.175+j 2.54$ & 0.403 & 0.069 \\
Optimized parameters & $-0.195+j 2.50$ & 0.398 & 0.078 \\
\hline
\end{tabular}

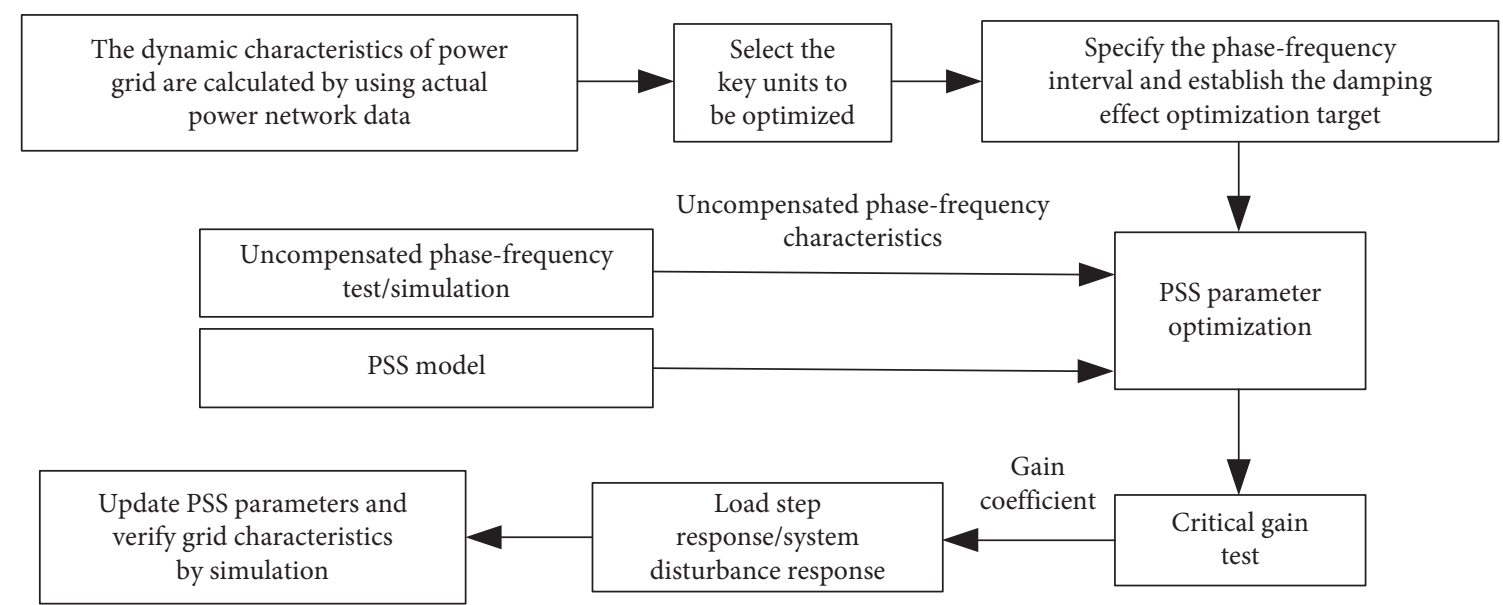

FIGURE 16: Flowchart for PSS parameters promotion suggested in this paper.

(4) Carry out optimization solution to obtain the PSS phase-shifting parameters

(5) Further determine the gain coefficient by the critical gain test

(6) Check whether the parameters meet the requirements of engineering standards and whether they have the appropriate damping effect

(7) Verify the improvement effect of the dynamic characteristics obtained by the power grid simulation analysis

\section{Conclusion}

In this paper, a comprehensive damping effect method is proposed to improve the PSS parameter optimization. The primary contribution/novelty of this work lies in that this method takes into account not only the phase-frequency characteristics but also amplitude-frequency properties to obtain the best optimizing effect.

The mathematical model of the proposed method is explained based on the analysis of the conventional phasefrequency method. In the proposed method, optimization objective is the maximum sum of the damping forces obtained in the target frequency range $(0-2 \mathrm{~Hz})$. Since this method improves the parameter optimization by both the phase-frequency and the amplitude-frequency aspects, it is more comprehensive and has a more satisfied effect.

The proposed method is compared with the engineering method, the existing optimization method, and the commercial software. The actual practical data from Northern Power Grid are employed for the comparison. It is shown that the PSS parameters obtained by the proposed method are superior to those obtained by the engineering experience, the phase-frequency optimization method, and the commercial software. In the cases of different phase-frequency range requirements, the wider the phase-frequency range is, the better the damping effect of the obtained parameters will be. Moreover, the proposed method can still achieve a better optimization effect even when the gain coefficient setting is taken into account.

The method proposed in this paper is able to improve the dynamic characteristics of multimachine systems and therefore is highly potential to be applied to improve the power limit of the transmission tie line.

\section{Data Availability}

The data used to support the findings of this study are available from the corresponding author upon request.

\section{Conflicts of Interest}

The authors declare that they have no conflicts of interest.

\section{Acknowledgments}

This work was supported in part by the Natural Science Foundation of China (no. 51777074), the Chinese Fundamental Research Funds for the Central Universities (no. 2018YQ03), and the Hebei Provincial Top Youth Talent Support Program ([2018]-27).

\section{References}

[1] K. Sebaa, H. Gueguen, and M. Boudour, "Mixed integer nonlinear programming via the cross-entropy approach for power system stabilisers location and tuning," IET Generation, Transmission \& Distribution, vol. 4, no. 8, pp. 928-939, 2010.

[2] T. Surinkaew and I. Ngamroo, "Coordinated robust control of DFIG wind turbine and PSS for stabilization of power 
oscillations considering system uncertainties," IEEE Transactions on Sustainable Energy, vol. 5, no. 3, pp. 823-833, 2014.

[3] A. Kumar, "Power system stabilizers design for multimachine power systems using local measurements," IEEE Transactions on Power Systems, vol. 31, no. 3, pp. 2163-2171, 2016.

[4] G. Tu, Y. Li, J. Xiang, and J. Ma, "Distributed power system stabiliser for multimachine power systems," IET Generation, Transmission \& Distribution, vol. 13, no. 5, pp. 603-612, 2019.

[5] D. Rimorov, A. Heniche, I. Kamwa et al., "Dynamic performance improvement of New York state power grid with multi-functional multi-band power system stabiliser-based wide-area control," IET Generation, Transmission \& Distribution, vol. 11, no. 18, pp. 4537-4545, 2017.

[6] M. Xiao-ming, Z. Yao, G. Lin, and W. Xiao-chen, "Coordinated control of interarea oscillation in the China southern power grid," IEEE Transactions on Power Systems, vol. 21, no. 2, pp. 845-852, 2006.

[7] O. Kahouli, M. Jebali, B. Alshammari, and H. Hadj Abdallah, "PSS design for damping low-frequency oscillations in a multi-machine power system with penetration of renewable power generations," IET Renewable Power Generation, vol. 13, no. 1, pp. 116-127, 2019.

[8] D. Ke and C. Y. Chung, "Design of probabilistically-robust wide-area power system stabilizers to suppress inter-area oscillations of wind integrated power systems," IEEE Transactions on Power Systems, vol. 31, no. 6, pp. 4297-4309, 2016.

[9] J. Zhang, L. Peng, and Q. Guo, "Negative damping case analysis and detection method of PSS2A model," Automation of Electric Power Systems, vol. 38, no. 2, pp. 127-130, 2014, in Chinese.

[10] N. Kulkarni, S. Kamalasadan, and S. Ghosh, "An integrated method for optimal placement and tuning of a power system stabilizer based on full controllability index and generator participation," IEEE Transactions on Industry Applications, vol. 51, no. 5, pp. 4201-4211, 2015.

[11] E. L. Miotto, P. B. de Araujo, E. de Vargas Fortes, B. R. Gamino, and L. F. B. Martins, "Coordinated tuning of the parameters of PSS and POD controllers using bioinspired algorithms," IEEE Transactions on Industry Applications, vol. 54, no. 4, pp. 3845-3857, 2018.

[12] Y. Liu, Q. H. Wu, H. Kang, and X. Zhou, "Switching power system stabilizer and its coordination for enhancement of multi-machine power system stability," CSEE Journal of Power and Energy Systems, vol. 2, no. 2, pp. 98-106, 2016.

[13] S. Guo, S. Zhang, W. Zhu, L. Li, and H. Li, "Power system stabiliser PSS4B-W parameters optimisation and RTDS test verification," The Journal of Engineering, vol. 2019, no. 16, pp. 3135-3139, 2019.

[14] D. Rimorov, I. Kamwa, and G. Joós, "Model-based tuning approach for multi-band power system stabilisers PSS4B using an improved modal performance index," IET Generation, Transmission \& Distribution, vol. 9, no. 15, pp. 21352143, 2015.

[15] X. Liu, Y. He, and J. Yao, "A new hybrid PSS optimization method based on improved active set algorithm," Mathematical Problems in Engineering, vol. 2018, Article ID 4348257, 9 pages, 2018.

[16] H. Verdejo, D. Gonzalez, J. Delpiano, and C. Becker, “Tuning of power system stabilizers using multiobjective optimization NSGA II," IEEE Latin America Transactions, vol. 13, no. 8, pp. 2653-2660, 2015.

[17] G. Sanchez-Ayala, V. Centeno, and J. Thorp, "Gain scheduling with classification trees for robust centralized control of
PSSs," IEEE Transactions on Power Systems, vol. 31, no. 3, pp. 1933-1942, 2016.

[18] A. I. Konara and U. D. Annakkage, "Robust power system stabilizer design using eigenstructure assignment," IEEE Transactions on Power Systems, vol. 31, no. 3, pp. 1845-1853, 2016.

[19] M. C. Pulcherio, M. S. Illindala, and R. K. Yedavalli, "Robust stability region of a microgrid under parametric uncertainty using bialternate sum matrix approach," IEEE Transactions on Power Systems, vol. 33, no. 5, pp. 5553-5562, 2018.

[20] National Energy Bureau, DL/T 1231-2013 Experiment Principles of PSS Setting, China Electric Power Press, Beijing, China, 2013.

[21] IEEE Standard, "IEEE recommended practice of excitation systems models for power system stability studies," IEEE Standard 421.5, 1992.

[22] IEEE Standard, "IEEE recommended practice for excitation system models for power system stability studies," IEEE Std. 421.5-2005.

[23] K. Wu, C. Lu, L. Wu et al., "A new PSS with double-signal input and its simulation research," Power System Technology, vol. 40, no. 5, pp. 1462-1468, 2016.

[24] I. Kamwa, R. Grondin, and G. Trudel, "IEEE PSS2B versus PSS4B: the limits of performance of modern power system stabilizers," IEEE Transactions on Power Systems, vol. 20, no. 2, pp. 903-915, 2005.

[25] C. Huo, Z. Liu, and F. Zhu, "Theory and field practice of phase compensation on generator excitation system by utilizing power system stabilizer," Proceedings of the CSEE, vol. 35, no. 12, pp. 2989-2997, 2015.

[26] Q. Liu, Power System Stability and Generator Excitation Control, China Electric Power Press, Beijing, China, 2007.

[27] P. K. Ray, S. R. Paital, A. Mohanty et al., "A hybrid fireflyswarm optimized fractional order interval type-2 fuzzy PIDPSS for transient stability improvement," IEEE Transactions on Industry Applications, vol. 55, no. 6, pp. 6486-6498, 2019.

[28] M. Liu, F. Bizzarri, A. M. Brambilla, and F. Milano, "On the impact of the dead-band of power system stabilizers and frequency regulation on power system stability," IEEE Transactions on Power Systems, vol. 34, no. 5, pp. 3977-3979, 2019.

[29] D. Chitara, K. R. Niazi, A. Swarnkar, and N. Gupta, "Cuckoo search optimization algorithm for designing of a multimachine power system stabilizer," IEEE Transactions on Industry Applications, vol. 54, no. 4, pp. 3056-3065, 2018.

[30] C. Xia, Z. Liu, F. Zhu, and H. Zhao, "Sectional compensation parallel power system stabilizer with two independent channels," Proceedings of the CSEE, vol. 30, no. 19, pp. 20-25, 2010, in Chinese. 